\title{
Petrov-Galerkin flux upwinding for mixed mimetic spectral elements, and its application to geophysical flow problems
}

\author{
David Lee ${ }^{\mathrm{a}, *}$ \\ ${ }^{a}$ Department of Mechanical and Aerospace Engineering, Monash University, Melbourne 3800, Australia
}

\begin{abstract}
Upwinded mass fluxes are described and analysed for advection operators discretised using mixed mimetic spectral elements. This involves a Petrov-Galerkin formulation by which the mass flux test functions are evaluated at downstream locations along velocity characteristics. As for the original mixed mimetic spectral element advection operator, the upwinded mass flux advection operator is conservative, however unlike the original advection operator, which is purely hyperbolic, the upwinded advection operator adds dissipation which is biased towards high wave numbers. The upwinded advection operator also removes the spectral gaps present in the dispersion relation for the original advection operator. As for the original advection operator, a material form advection operator may be constructed by similarly downwinding the trial functions of the tracer gradients. Both methods allow for the recovery of exact energy conservation for an incompressible flow field via skew-symmetric formulations. However these skew-symmetric formulations are once again purely hyperbolic operators which do not suppress oscillations. The scheme is implemented within a shallow water code on the sphere in order to diagnose and interpolate the potential vorticity. In the absence of other dissipation terms, it is shown to yield more coherent results for a standard test case of barotropic instability.
\end{abstract}

Keywords: Advection, Upwinding, Mimetic, Compatible, Mixed finite elements,

It is well known that in the absence of upwinding, diffusion or other stabilisation schemes, discrete Eulerian approximations to hyperbolic terms result in spurious oscillations. Numerous methods have been devised to address this issue in the context of finite element methods, including the streamwise-upwind Petrov-Galerkin method [1] and variational multiscale methods [2]. These schemes have subsequently been applied within collocated spectral element discretisations [3]. In the present work we address this issue in the context of a mixed mimetic spectral element discretisation [4], a high order finite element method for which the $H(\operatorname{div}, \Omega)$ function space of the mass flux is compatibly mapped to the $L^{2}(\Omega)$ space of the tracer field by the divergence operator [5].

Existing variational approaches to upwind stabilisation typically involve augmenting the test space with additional terms, for example a convective term that results in some form of symmetric positive definite operator which acts as a viscosity in the direction of the flow [1], or additionally a dual space in which the fine scales are represented via an adjoint problem from which the stabilisation terms are derived [2]. An alternative form of upwinding for mixed finite elements for Hamiltonian systems has been introduced whereby all of the test and trial functions in the $H(\operatorname{div}, \Omega)$ space are projected onto upwind variants within the skew-symmetric operator so as to preserve energy conservation [6]. Here we instead base our methods around the idea of Lagrangian basis functions, which are evaluated at upstream locations in order to stabilise the resultant mass fluxes.

Mass conserving variational schemes based on Lagrangian basis functions have been developed previously for both finite element [7] and discontinuous Galerkin [8-10] methods. In each of the above cases the computation of fluxes via the extrusion of the test functions over the tracer field implies an adjoint problem in which the test functions are themselves advected along Lagrangian characteristics. In the present mixed finite element context, only the test functions for the mass fluxes are advected, and mass conservation is ensured since the trial functions, which compatibly map to the trial functions for the tracer via the divergence operator, remain static.

\footnotetext{
${ }^{*}$ Corresponding author. Tel. +61 452262804

Email address: davelee2804@gmail .com (David Lee)
} 
In the following section the advection problem is introduced for the standard mixed mimetic spectral element discretisation. In Section 2 variations on this method using upwinded test functions for flux form advection, or downwinded trial functions for material form advection will be described. Results for these new formulations, and comparisons to the original scheme will be presented in Section 3. In Section 4 the scheme is extended to two dimensions by way of some standard test cases for passive advection on the sphere. As a practical demonstration of the Petrov-Galerkin upwind stabilisation, results are presented for the diagnosis and interpolation of potential vorticity for the rotating shallow water equations on the sphere in Section 5, and conclusions are discussed in Section 6.

\section{Advection using mixed mimetic spectral elements}

Consider the scalar advection equation of a tracer, $q$, subject to a velocity field, $\boldsymbol{u}$, expressed in conservative flux form as

$$
\begin{aligned}
\dot{q}+\nabla \cdot \boldsymbol{F} & =0, \\
\boldsymbol{F} & =\boldsymbol{u} q,
\end{aligned}
$$

where $\boldsymbol{F}$ is the mass flux, within a periodic spatial domain $\Omega=[0, L) \subset \mathbb{R}$, and a temporal coordinate $t=[0, \infty)$. We further assume the existence of two discrete, finite dimensional subspaces, $\mathcal{U}_{h} \subset H(\operatorname{div}, \Omega)$ and $Q_{h} \subset L^{2}(\Omega)$, such that $Q_{h}$ contains a set of functions which are square integrable, and $\mathcal{U}_{h}$ contains a set of functions for which the sum of the functions and their divergence are square integrable. These spaces satisfy a compatibility property of the form

$$
\mathcal{U}_{h} \stackrel{\nabla \cdot}{\rightarrow} Q_{h}
$$

These two discrete subspaces are composed of a finite set of polynomial basis functions of degree $p$ within each element such that

$$
\mathcal{U}_{h}=\operatorname{span}\left\{l_{0}^{p}(\xi), \ldots, l_{p}^{p}(\xi)\right\}, \quad Q_{h}=\operatorname{span}\left\{e_{0}^{p}(\xi), \ldots, e_{p-1}^{p}(\xi)\right\}
$$

where $\xi$ is a local coordinate within the canonical element domain $[-1,1] \subset \mathbb{R}$. For the remainder of this article, we will use as these subspaces the Lagrange polynomials of degree $p$ with their roots as the Gauss-Lobatto-Legendre (GLL) points of equal degree, and the associated edge functions [4] respectively. These are given for the GLL nodes $\xi_{k}$ as

$$
l_{i}^{p}(\xi)=\prod_{\substack{k=0 \\ k \neq i}}^{p} \frac{\xi-\xi_{k}}{\xi_{i}-\xi_{k}}, \quad e_{i}^{p}(\xi)=-\sum_{k=0}^{i-1} \frac{\mathrm{d} l_{k}^{p}(\xi)}{\mathrm{d} \xi} .
$$

The discrete mass flux, $\boldsymbol{F}_{h} \in \mathcal{U}_{h}$, and tracer field, $q_{h} \in Q_{h}$ may be interpolated via the nodal and edge bases respectively as

$$
\boldsymbol{F}_{h}(\xi)=\sum_{i=0}^{p} \hat{F}_{i} l_{i}^{p}(\xi), \quad q_{h}(\xi)=\sum_{i=0}^{p-1} \hat{q}_{i} e_{i}^{p}(\xi),
$$

where $\hat{F}_{i}$ and $\hat{q}_{i}$ are the degrees of freedom. For the remainder of this article we will drop the superscripts, $l_{i}^{p}(\xi)$ and $e_{i}^{p}(\xi)$, and assume that $p$ remains fixed at some specified degree for a given configuration.

Due to the orthogonality and compatibility properties of $\mathcal{U}_{h}$ and $Q_{h}$, by which the fundamental theorem of calculus is satisfied exactly between GLL (or any other choice of) nodes, the discrete divergence operator may be represented by a purely topological relation, known as an incidence matrix [5], and defined here over a periodic one dimensional domain as

$$
\mathbf{E}=\left[\begin{array}{cccccc}
-1 & 1 & 0 & 0 & \ldots & 0 \\
0 & -1 & 1 & 0 & \ldots & 0 \\
0 & 0 & -1 & 1 & \ldots & 0 \\
\vdots & \vdots & \vdots & \vdots & \ddots & \vdots \\
1 & 0 & 0 & 0 & \ldots & -1
\end{array}\right]
$$

The incidence matrix provides a strong form mapping between degrees of freedom in $\mathcal{U}_{h}$ and those in $Q_{h}$, and as such is a discrete representation of (2). 
The discrete divergence of the mass flux onto a semi-discrete time derivative of the tracer field is then given in the strong form as

$$
\dot{\hat{q}}_{i}=-\mathrm{E}_{i j} \hat{F}_{j} .
$$

We wish to derive a mass flux that is $C^{0}$ continuous across element boundaries, and so compute this via a contraction of the tracer field onto the velocity field as

$$
\left\langle l_{i}, l_{j}\right\rangle_{\Omega} \hat{F}_{j}=\left\langle l_{i} \cdot \boldsymbol{u}_{h}, e_{k}\right\rangle_{\Omega} \hat{q}_{k}, \quad \forall l_{i} \in \mathcal{U}_{h},
$$

where the brackets $\langle a, b\rangle_{\Omega}=\int a b \mathrm{~d} \Omega$ correspond to a bilinear operator and the subscript $\Omega$ denotes the integration and assembly over all elements in the domain $\Omega$. Note that the set of nodal basis functions $l_{i}(\xi)$ have the same representation in both local and global coordinates, and so the transformation of these bases to global coordinates is unity, and does not involve the Jacobian, while the transformation for $e_{i}(\xi) \in Q_{h}$ is the inverse of the Jacobian determinant, $|J(\xi)|^{-1}$. In higher dimensions these are transformed via the Piola mappings [11, 12].

Equations (7) and (8) describe the semi-discrete integration of (1) [13, 14]. These may be expressed in a single equation as

$$
\left\langle e_{i}, e_{j}\right\rangle_{\Omega} \dot{\hat{q}}_{j}+\left\langle e_{i}, e_{k}\right\rangle_{\Omega} \mathrm{E}_{k m}\left\langle l_{n}, l_{m}\right\rangle_{\Omega}^{-1}\left\langle l_{n} \cdot \boldsymbol{u}_{h}, e_{r}\right\rangle_{\Omega} \hat{q}_{r}=0 \quad \forall e_{i} \in Q_{h},
$$

where both sides of (7) have been pre-multiplied by the $Q_{h}$ mass matrix, $\mathbf{M}=\left\langle e_{i}, e_{j}\right\rangle_{\Omega}$. This equation conserves mass due to the telescopic property of the incidence matrix, $\mathbf{1}^{\top} \mathbf{E}=\mathbf{0}[14]$. Note that in the multi-dimensional case the discrete subspace of $H(\operatorname{div}, \Omega)$ is composed of vector functions with continuous normal components, and so $l_{i}$ is replaced with a vector field basis with $C^{0}$ continuity across element boundaries.

Notably, 97 is the adjoint of the discrete material form of the advection equation

$$
\begin{aligned}
\dot{q}+\boldsymbol{u} \cdot \boldsymbol{G} & =0, \\
\boldsymbol{G} & =\nabla q,
\end{aligned}
$$

where $\boldsymbol{G}$ is the tracer gradient. The degrees of freedom of the discrete form of the tracer gradient, $\hat{G}_{i}$, are determined via a weak form integration by parts relation with respect to the strong form divergence operator (assuming periodic boundary conditions) as [15]

$$
\hat{G}_{j}=-\left\langle l_{i}, l_{j}\right\rangle_{\Omega}^{-1} \mathrm{E}_{k, i}^{\top}\left\langle e_{k}, e_{l}\right\rangle \hat{q}_{l} \quad \forall l_{i} \in \mathcal{U}_{h} .
$$

The discrete variational form of $(10)$, combined with 111 then gives the discrete form of the material advection equation as

$$
\left\langle e_{i}, e_{j}\right\rangle_{\Omega} \dot{\hat{q}}_{j}-\left\langle e_{i}, \boldsymbol{u}_{h} \cdot l_{k}\right\rangle_{\Omega}\left\langle l_{m}, l_{k}\right\rangle_{\Omega}^{-1} \mathrm{E}_{n m}^{\top}\left\langle e_{n}, e_{r}\right\rangle_{\Omega} \hat{q}_{r}=0 \quad \forall e_{i} \in Q_{h} .
$$

The adjoint property is then satisfied since the advection operator in (9),

$$
\mathbf{A}=\left\langle e_{i}, e_{k}\right\rangle_{\Omega} \mathrm{E}_{k m}\left\langle l_{n}, l_{m}\right\rangle_{\Omega}^{-1}\left\langle l_{n} \cdot \boldsymbol{u}_{h}, e_{r}\right\rangle_{\Omega},
$$

and the corresponding operator in (12),

$$
\mathbf{B}=-\left\langle e_{i}, \boldsymbol{u}_{h} \cdot l_{k}\right\rangle_{\Omega}\left\langle l_{m}, l_{k}\right\rangle_{\Omega}^{-1} \mathrm{E}_{n m}^{\top}\left\langle e_{n}, e_{r}\right\rangle_{\Omega},
$$

are related as $\mathbf{B}=-\mathbf{A}^{\top}$.

As with other discretisations [16, 17], the adjoint property for the flux form and material form advection operators allows for exact energy conservation for incompressible flows $\left(\mathbf{E} \hat{\boldsymbol{u}}_{h}=\mathbf{0}, \forall \boldsymbol{u}_{h} \in \mathcal{U}_{h}\right)$. This is achieved via a skewsymmetric form of the advection operator as

$$
\mathbf{S}=\frac{1}{2}(\mathbf{A}+\mathbf{B})=\frac{1}{2}\left(\mathbf{A}-\mathbf{A}^{\top}\right)
$$

and a centered time integration scheme of the form

$$
\mathbf{M} \frac{\left(\hat{q}_{h}^{n+1}-\hat{q}_{h}^{n}\right)}{\Delta t}+\mathbf{S} \frac{\left(\hat{q}_{h}^{n+1}+\hat{q}_{h}^{n}\right)}{2}=0 .
$$

Pre-multiplying both sides of $(16)$ by $\left(\hat{q}_{h}^{n+1}+\hat{q}_{h}^{n}\right) / 2$ gives the conservation of energy as

$$
\hat{q}_{h}^{n+1} \mathbf{M} \hat{q}_{h}^{n+1}=\hat{q}_{h}^{n} \mathbf{M} \hat{q}_{h}^{n},
$$

due to the skew-symmetry of $\mathbf{S}$ and the bi-linearity of $\mathbf{M}$. 


\section{Petrov-Galerkin flux upwinding}

The advection operators described in the preceeding section have numerous appealing properties, including high order error convergence (for smooth solutions), mass and energy conservation, and purely imaginary eigenvalues (strict hyperbolicity). However they are also prone to spurious oscillations in the presence of sharp, poorly resolved gradients. In this section we describe modified formulations which smooth out these oscillations via upwinding. A consequence of this upwinding construction is that the eigenvalues of the operators are no longer purely hyperbolic, such that non-zero real eigenvalues are present that act to damp the solutions.

In order to upwind the mass flux test functions, we may equivalently evaluate these at downstream locations [9], defined locally as

$$
\xi^{d}=\xi+\Delta t \int_{s=0}^{1}|J(\xi(s))|^{-1} \boldsymbol{u}(\xi(s), s) \mathrm{d} s,
$$

where $|J(\xi(s))|^{-1}$ is the Jacobian determinant inverse and $\boldsymbol{u}(\xi(s), s)$ is the velocity in local element coordinates, such that $l_{i}^{u}=l_{i}\left(\xi^{d}\right)$. The physical units of the time step and the Jacobian determinant are introduced in order to ensure that the amount of upwinding is small with respect to the flow velocity. High order basis functions in $\mathcal{U}_{h}$ diverge in proportion to their polynomial degree outside of the canonical element domain, and so if these are upwinded excessively then the condition number of the matrix will degrade significantly. The choice of upwinding distance used here works well experimentally but is not unique. Just as the traditional SUPG method [1] is dependent on the choice of a tuning parameter, there is perhaps some way to optimise the upwinding length scale here also.

The mass flux is then computed using a Petrov-Galerkin formulation as

$$
\left\langle l_{i}^{u}, l_{j}\right\rangle_{\Omega} \hat{F}_{j}^{P G}=\left\langle l_{i}^{u} \cdot \boldsymbol{u}_{h}, e_{k}\right\rangle_{\Omega} \hat{q}_{k}, \quad \forall l_{i}^{u} \in \mathcal{U}_{h} .
$$

Since the trial space remains unaltered, the resulting mass flux $\hat{F}_{j}^{P G}$ maintains its compatible mapping with respect to the space of the tracer field, $Q_{h}$, such that mass conservation is preserved for the upwinded flux. The choice to upwind the test functions for the mass flux and not the full advection equation, as is customary in Petrov-Galerkin formulatons [1, 2], is motivated by the need to upwind a test space for which $C^{0}$ continuity is enforced in the direction of the flow. If the tests function for the full advection equation $(9$ ) are upwinded instead of those for the mass flux, then nothing is gained, since these are discontinuous across element boundaries, and moreover the $Q_{h}$ mass matrix $\mathbf{M}$ can be cancelled from (9) since the divergence operator is expressed in the strong form. The Petrov-Galerkin upwinded advection operator is then given as

$$
\mathbf{A}_{P G ; \Delta t}=\left\langle e_{i}, e_{k}\right\rangle_{\Omega} \mathrm{E}_{k m}\left\langle l_{n}^{u}, l_{m}\right\rangle_{\Omega}^{-1}\left\langle l_{n}^{u} \cdot \boldsymbol{u}_{h}, e_{r}\right\rangle_{\Omega} .
$$

The corresponding material form advection operator is given as

$$
\mathbf{B}_{P G ; \Delta t}=-\mathbf{A}_{P G ;-\Delta t}^{\top}=-\left\langle e_{i}, \boldsymbol{u}_{h} \cdot l_{k}^{d}\right\rangle_{\Omega}\left\langle l_{m}, l_{k}^{d}\right\rangle_{\Omega}^{-1} \mathrm{E}_{n m}^{\top}\left\langle e_{n}, e_{r}\right\rangle_{\Omega}
$$

where $l_{i}^{d}=l_{i}\left(\xi^{u}\right)$ and

$$
\xi^{u}=\xi-\Delta t \int_{s=0}^{1}|J(\xi(s))|^{-1} \boldsymbol{u}(\xi(s), s) \mathrm{d} s,
$$

Note that in each of the examples presented in this article we use a simple first order Euler integration in order to determine $\xi^{d}$ and $\xi^{u}$. For multi-dimensional flows involving large amounts of deformation, it may be advisable to use higher order integration in order to determine the departure locations of the Gauss-Lobatto quadrature points. No assumption has been made about $\boldsymbol{u}_{h}$ in either (20) or (21), other than this be uniquely defined and that it represents a vector field for multi-dimensional domains. As such the velocity field may be either compressible or incompressible. The only instance in which incompressibility is required is in the construction of a skew-symmetric advection operator, as in (15).

One drawback of the upwinded operators described above is that by evaluating oscillatory basis functions outside of the canonical domain the condition number of the matrices necessary to determine mass fluxes and tracer gradients is increased. Also for nodal bases which are orthogonal with respect to the standard Gauss-Lobatto quadrature points, by moving these quadrature point locations this orthogonality is broken such that the corresponding mass matrices are no longer diagonal. 

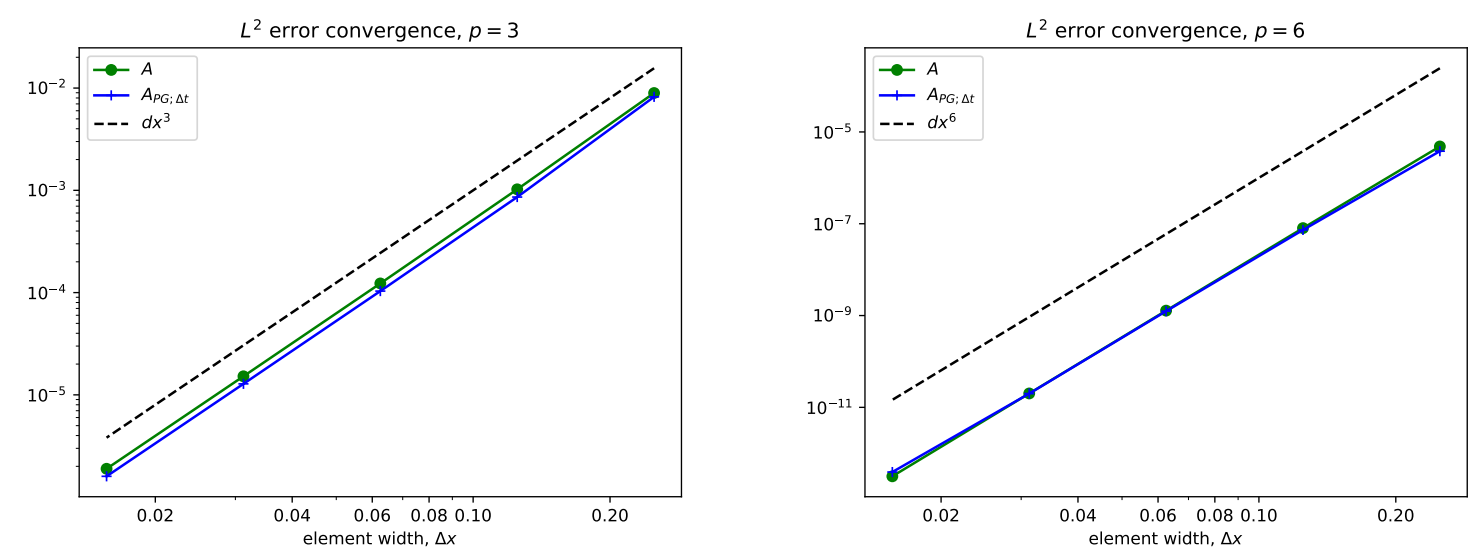

Figure 1: Mass flux error convergence for $p=3$ (left) and $p=6$ (right).
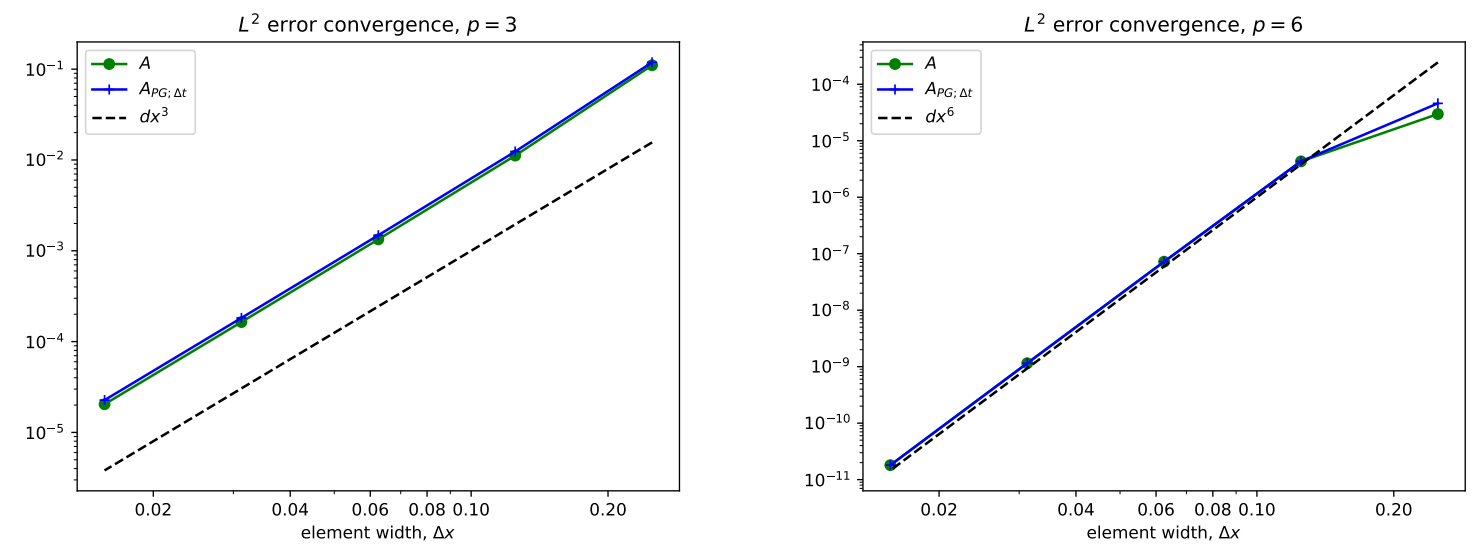

Figure 2: Material advection operator error convergence for $p=3$ (left) and $p=6$ (right).

\section{Results}

We first verify the error convergence properties for the upwinded mass flux, for a manufactured solution of the form $q=0.5(1.0-\cos (2 \pi x)), \boldsymbol{u}=0.4+0.2(1.0+\sin (2 \pi x))$ over a periodic domain of unit length $L=1$, such that $x \in[0, L)$, and compare the convergence rates against the original definition of the discrete mass flux for $\boldsymbol{F}=\boldsymbol{u} q$. For this test we compare two configurations, the first for elements of degree $p=3$, and the second for elements of degree $p=6$. In both cases we use $n_{e}=4 \times 2^{n}$ elements, with $n=1,2,3,4,5$ and a time step of $\Delta t=0.1 / n_{e}$ in order to upwind the trial functions for the Petrov-Galerkin formulation. As observed in Fig. 1. both the original and upwinded mass fluxes converge at their theoretical rates for both $p=3$ and $p=6$ within the $L^{2}(\Omega)$ norm. The convergence is marginally better for the upwinded formulation, however this improvement diminishes with polynomial order.

The error convergence of the material form advection operator (14) and its downwinded variant (21) is then verified against a manufactured solution with the same specification of $q$ and $\boldsymbol{u}$ as for the previous test case. For polynomials of degree $p=3$ and $p=6$, the errors converge at the expected rate for both formulations, as shown in Fig. (2).

As a second test we time step the advection equation using the original, upwinded flux form, and downwinded material form advection operators for an incompressible flow of $\boldsymbol{u}=0.4$ and an initial tracer distribution of

$$
q(x, 0)= \begin{cases}0.5+0.5 \tanh (200(x-0.4)), & x<0.5 \\ 0.5+0.5 \tanh (200(0.6-x)), & x \geq 0.5\end{cases}
$$



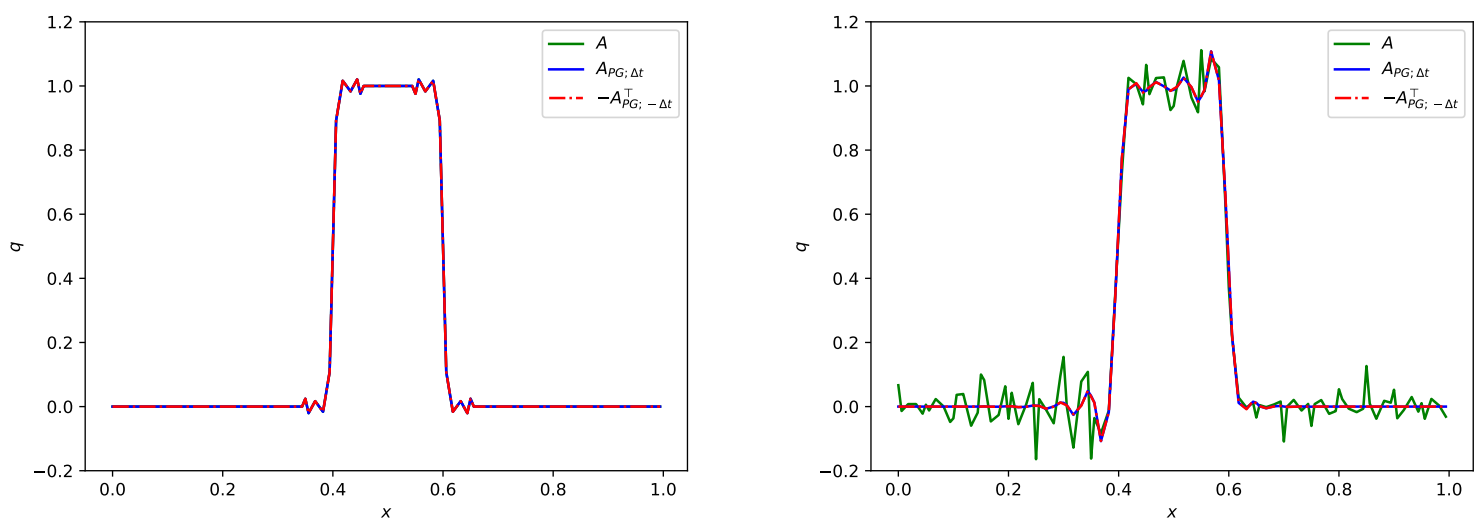

Figure 3: Initial state (left) and final state (right) after one revolution, $p=5,20$ elements, $u=0.4, \Delta t=0.005$.
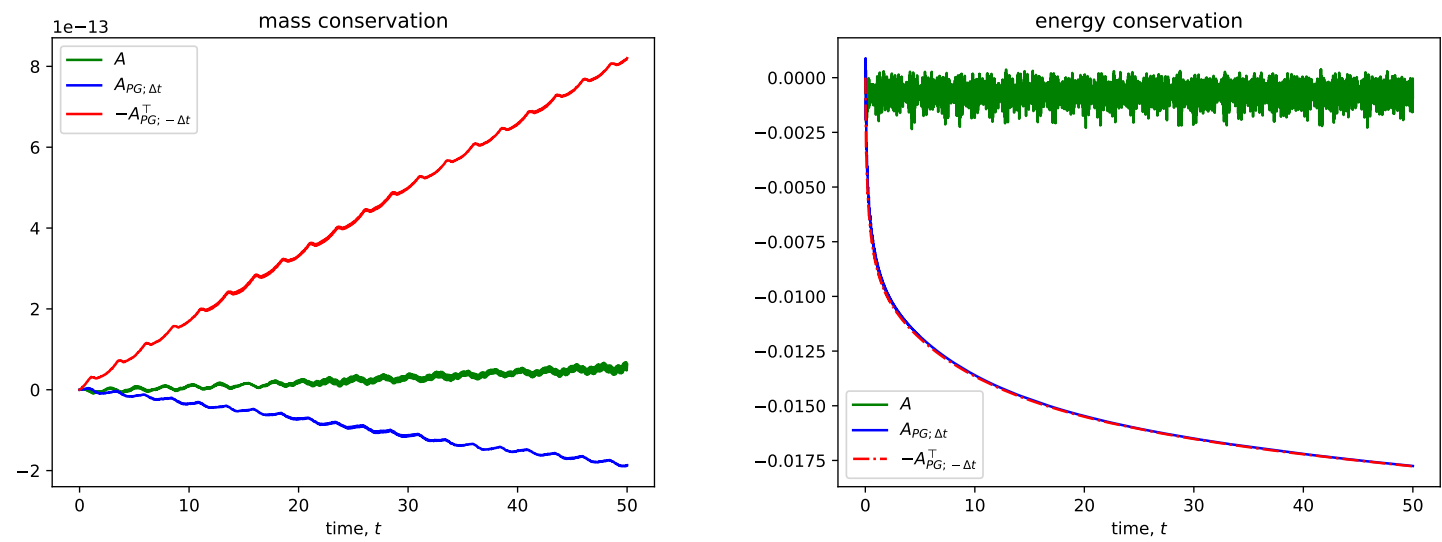

Figure 4: Mass (left) and energy (right) conservation errors with time, $p=5,20$ elements, $u=0.4, \Delta t=0.005$.

over the unit domain of $L=1$ with 20 elements and a time step of $\Delta t=0.005$ over a single revolution of period $T=2.5$. In each case a second order centered time stepping scheme of the form

$$
\mathbf{M} \frac{\left(\hat{q}_{h}^{n+1}-\hat{q}_{h}^{n}\right)}{\Delta t}+\mathbf{A} \frac{\left(\hat{q}_{h}^{n+1}+\hat{q}_{h}^{n}\right)}{2}=0
$$

was employed (with $\mathbf{A}$ replaced with $\mathbf{A}_{P G ; \Delta t}$ and $-\mathbf{A}_{P G ;-\Delta t}^{\top}$ for the upwinded flux form and downwinded material forms respectively).

Figure 3 shows the initial and final results for the advection test. Note that the initial data is somewhat oscillatory since this is the projection of an analytical function with sharp gradients onto the $Q_{h}$ basis functions for which continuity is not enforced across element boundaries. The upwinded test function flux form and downwinded trial function material form solutions are indistinguishable, however both are markedly less oscillatory than the original solution. The corresponding mass and energy conservation errors over 20 revolutions are given in Fig. 4 A small drift in mass conservation away from machine precision is perceptible for all three schemes, but is somewhat greater for the upwinded schemes. Since this is present for the original advection operator, $\mathbf{A}$ (13), which is known to conserve mass [14], we surmise that this drift is due to the time stepping scheme. While the energy conservation errors remain bounded for the original advection operator, the Petrov-Galerkin operators dissipate energy. Note that $\mathbf{A}$ as used in 24) is not itself skew-symmetric, so exact energy conservation is not anticipated. 

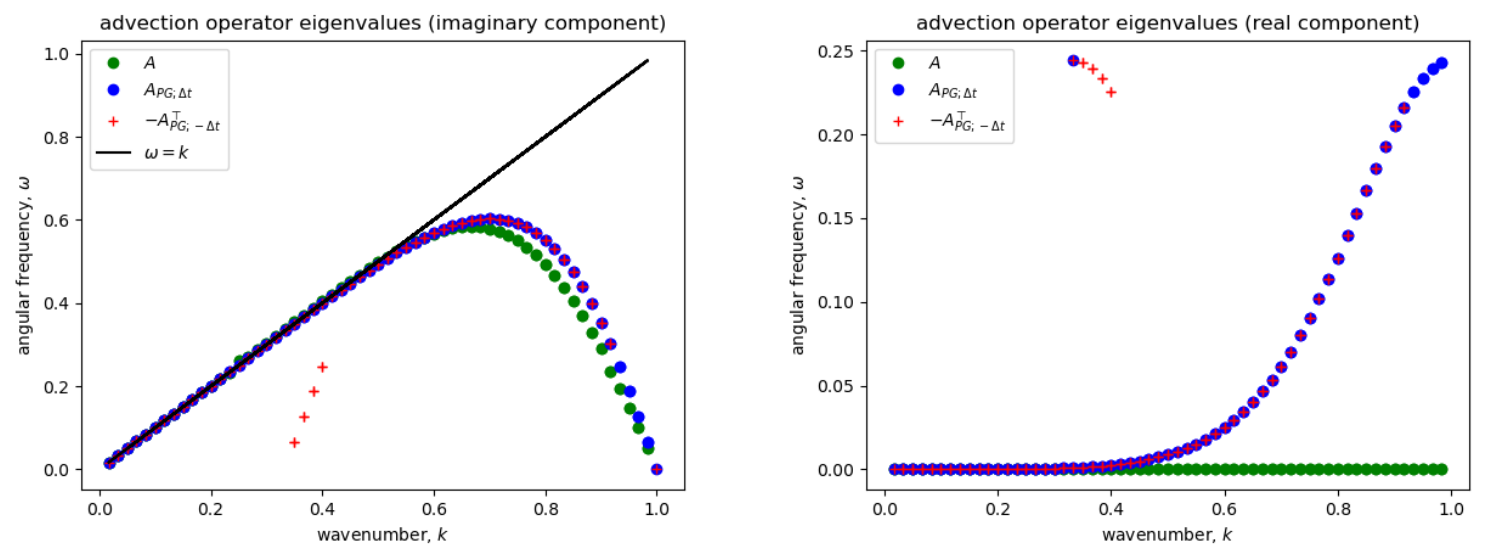

Figure 5: Imaginary (left) and real (right) eigenvalues for the advection operators, 40 elements, $p=3, u=0.4, \Delta t=0.005$.
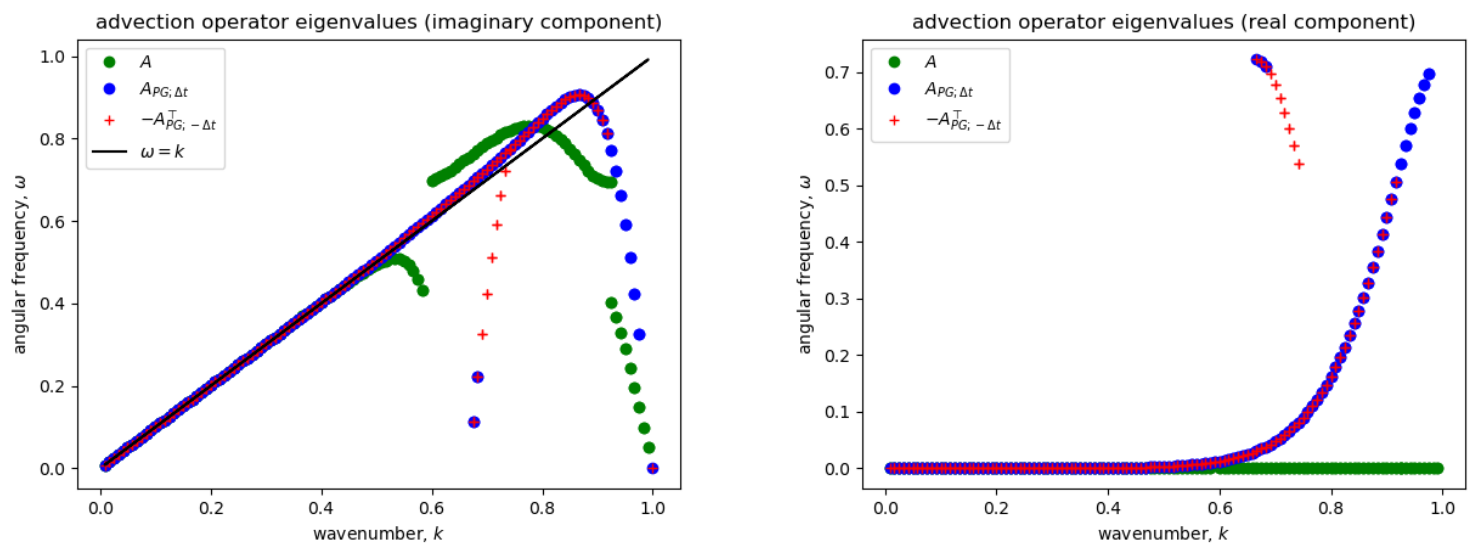

Figure 6: Imaginary (left) and real (right) eigenvalues for the advection operators, 40 elements, $p=6, u=0.4, \Delta t=0.005$.

Figures 5 and 6 show the imaginary and real components of the dispersion relations for the advection operators of degree $p=3$ and $p=6$ respectively with 40 elements and a time step of $\Delta t=0.005$. These are computed by first interpolating the eigenvectors from the $Q_{h}$ space to physical space, and then projecting these physical space solutions onto Fourier modes (for details see the appendix).

The imaginary eigenvalues of the Petrov-Galerkin operators are closer to the analytic, dispersionless solution of $\omega=k$ (indicated by the solid black lines in Figs. 5 and 6). Notably the Petrov-Galerkin operators also seal up the spectral gaps which are characteristic of both collocated [18] and mixed [19] high order finite element discretisations. This behaviour has also been observed for hyperviscosity in the context of collocated spectral elements [20]. However while the real eigenvalues are at machine precision for the original advection operator, $\mathbf{A}$, indicating purely hyperbolic advection, these are non-zero for the Petrov-Galerkin formulations, indicating dissipative solutions biased towards higher wave numbers. The dissipation profile of these operators steepens with polynomial degree, in analogy to a higher power viscosity operator.

Figure 7 shows the real component of the eigenvalues, $\omega^{r}$ against the imaginary, $\omega^{i}$ for the centered time stepping operator $(\mathbf{M}+0.5 \Delta t \mathbf{A})^{-1}(\mathbf{M}-0.5 \Delta t \mathbf{A})$ (and similarly for $\mathbf{A}_{P G ; \Delta t}$ and $-\mathbf{A}_{P G ;-\Delta t}^{\top}$ ), as given in (24), for a time step of $\Delta t=0.005$. The eigenvalues determined by evaluating the time stepping operator with respect to $\mathbf{A}$ sit on the unit circle, indicating the neutral stability of this formulation. This is also reflected in the bounded energy conservation errors, as shown in Fig. 4. The dissipative nature of the Petrov-Galerkin operators is reflected by the fact that the 

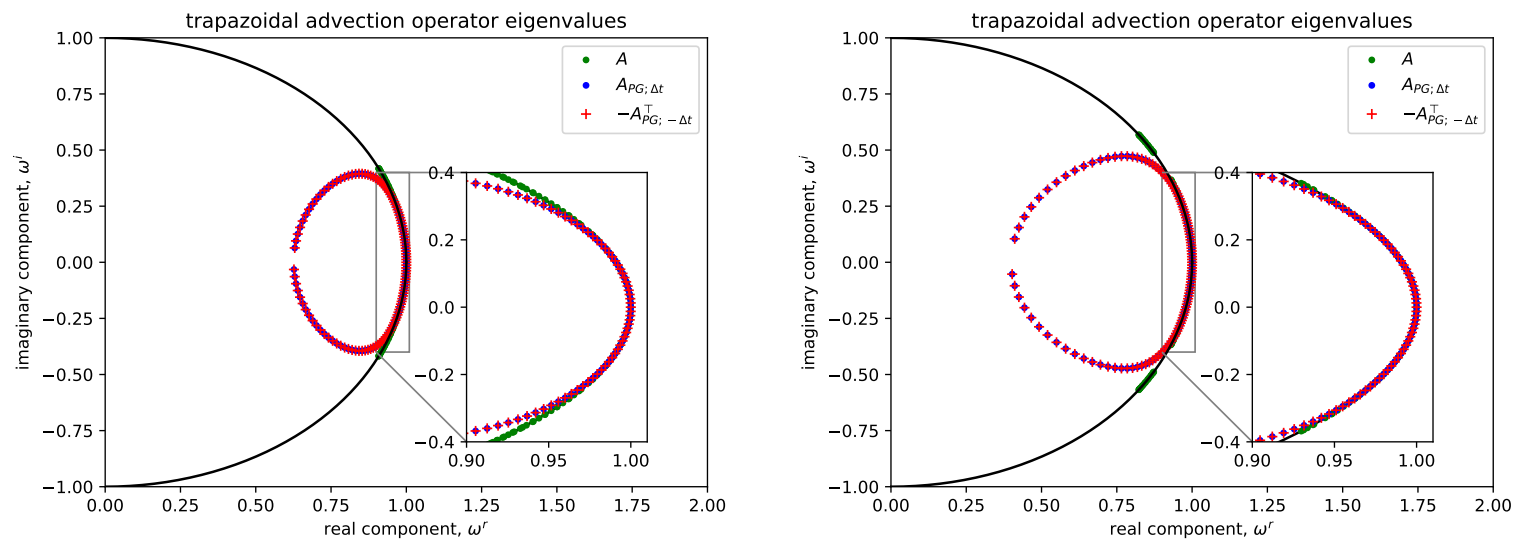

Figure 7: Time centered advection operator eigenvalues (real), $p=3,40$ elements (left), and $p=6,20$ elements (right), $u=0.4, \Delta t=0.005$. The black line indicates the unit circle.
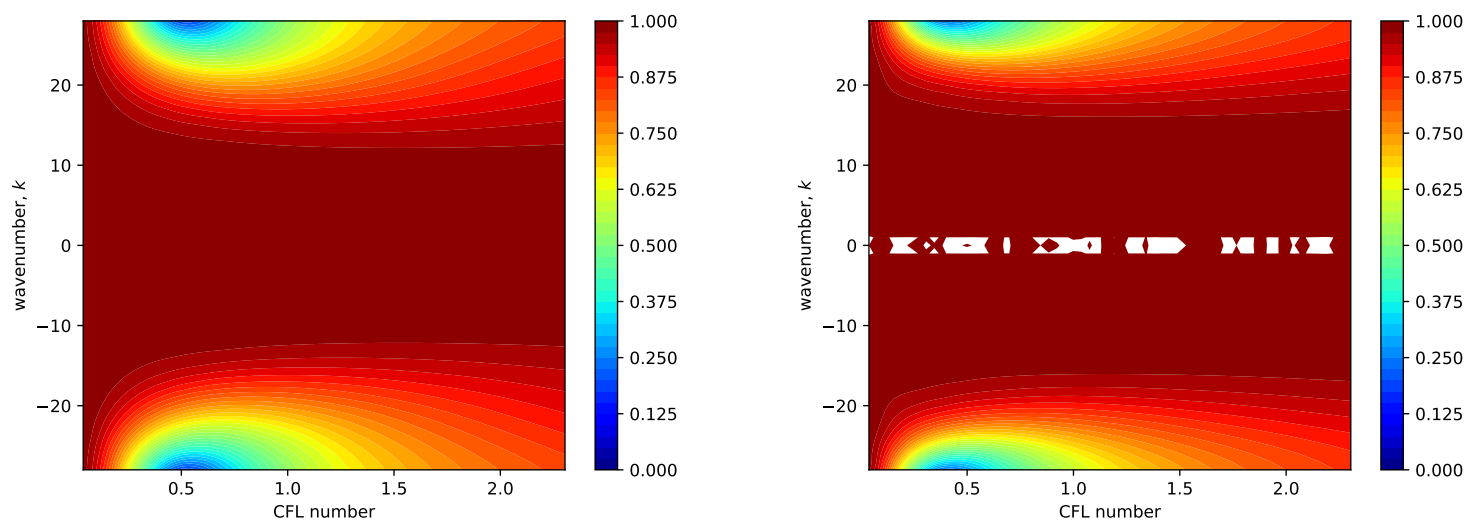

Figure 8: Absolute eigenvalue of the time centered flux form Petrov-Galerkin advection operator, $|\omega|$, as a function of CFL number, $\Delta t|\boldsymbol{u}| n_{e} p / L$ and Fourier wavenumber, $k ; p=3, n_{e}=20$ (left) and $p=6, n_{e}=10$ (right).

eigenvalues for the time integration operator sit inside the unit circle.

The A-stability of the upwinded flux form time stepping operator, $\left(\mathbf{M}+0.5 \Delta t \mathbf{A}_{P G ; \Delta t}\right)^{-1}\left(\mathbf{M}-0.5 \Delta t \mathbf{A}_{P G ; \Delta t}\right)$, is determined from the magnitude of its eigenvalues, $|\omega|$. These are plotted against an approximate CFL number, $\Delta t|\boldsymbol{u}| n_{e} p / L$ (which does not account for the smaller distance between the GLL nodes near the element boundaries), and Fourier wavenumber, $k$ for polynomials of degree $p=3$ and $p=6$ in Fig. 8 . While dissipation increases for larger absolute wavenumbers $|k|$, as indicated by smaller values of $|\omega|$, it is greatest for moderate CFL numbers. Since the time centered advection operator is unconditionally stable, nowhere is $|\omega|>1$. Additionally, for larger CFL numbers the upwinded operators fail to preserve their theoretical rate of convergence.

As for the original advection operator, A, both forms of the Petrov Galerkin advection operator may be used to construct skew-symmetric formulations, as given in (15). In each case there is a small drift away from machine precision in the energy conservation as shown in Fig. 9, which is also observed for the mass conservation in Fig. 4 and is due to the mass conservation errors in the time stepping scheme. These skew-symmetric formulations directly result from the cancellation of the upwinding contributions of the Petrov-Galerkin operators such that the solutions are once again oscillatory and there is no discernible benefit to the use of these formulations over the original advection operator. 

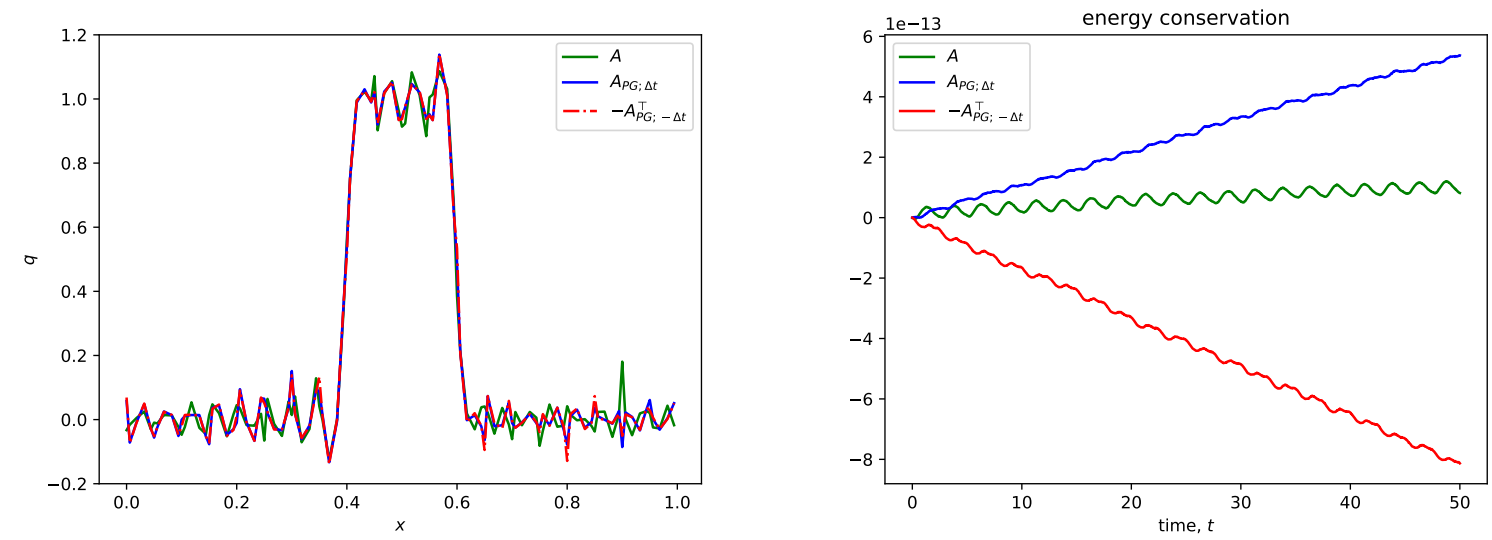

Figure 9: Skew-symmetric formulation: final state after one revolution (left) and energy conservation errors (right), $p=5,20$ elements, $u=0.4$, $\Delta t=0.005$.

\section{Extension to multiple dimensions: Advection on the sphere}

Having validated the new advection scheme for the one dimensional case, the scheme is extended to the case of passive advection on the surface of the sphere. The compatibility property between the discrete spaces $U_{h} \subset$ $H\left(\operatorname{div}, \Omega^{2}\right)$ and $Q_{h} \subset L^{2}\left(\Omega^{2}\right)(2)$ is also satisfied in the two dimensional domain, $\Omega^{2} \subset \mathbb{R}^{2}$, as is the strong form mapping of the incidence matrix, $\mathbf{E}^{2,1}$ between these spaces and the weak form adjoint relationship to the discrete gradient operator (11). However in multiple dimensions the bases that span these function spaces are constructed from tensor product combinations of the nodal and edge polynomials (4). These are given respectively for the two dimensional basis functions $\boldsymbol{\beta}_{h} \in U_{h}$ and $\gamma_{h} \in Q_{h}$ for polynomial degree $p$ as

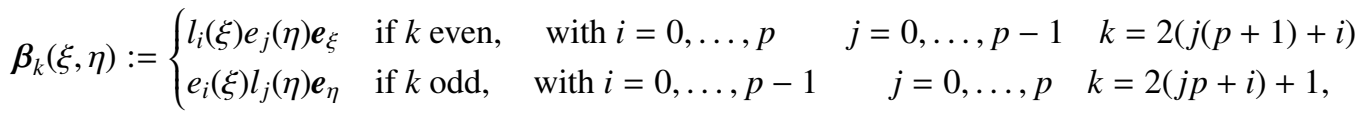

$$
\begin{aligned}
& \gamma_{k}(\xi, \eta):=e_{i}(\xi) e_{j}(\eta), \quad i, j=0, \ldots, p-1 \quad k=j p+i \text {. }
\end{aligned}
$$

for which

$$
U_{h}=\operatorname{span}\left\{\boldsymbol{\beta}_{0}(\xi, \eta), \ldots, \boldsymbol{\beta}_{2 p(p+1)-1}(\xi, \eta)\right\}, \quad Q_{h}=\operatorname{span}\left\{\gamma_{0}(\xi, \eta), \ldots, \gamma_{p \times p-1}(\xi, \eta)\right\},
$$

where $(\xi, \eta)$ are the local coordinates in the dimensions $\boldsymbol{e}_{\xi}, \boldsymbol{e}_{\eta}$ respectively within the two dimensional canonical domain $[-1,1] \times[-1,1]$.

In order to upwind the $U_{h}$ bases, these are evaluated at downstream locations according to (18) only in dimensions for which $C^{0}$ continuity is enforced, such that the upwinded form of 25a is given as

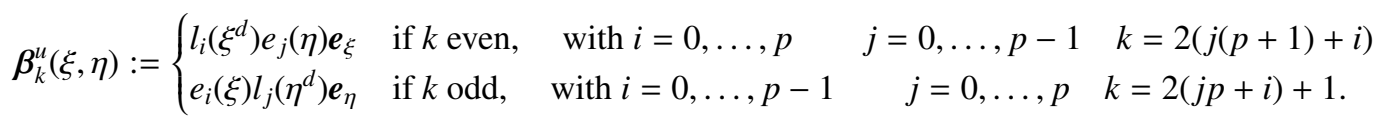

The two dimensional upwinded flux form advection equation is then given as

$$
\left\langle\gamma_{i}, \gamma_{j}\right\rangle_{\Omega^{2}} \dot{\hat{q}}_{j}+\left\langle\gamma_{i}, \gamma_{k}\right\rangle_{\Omega^{2}} \mathrm{E}_{k, l}^{2,1}\left\langle\boldsymbol{\beta}_{m}^{u}, \boldsymbol{\beta}_{l}\right\rangle_{\Omega^{2}}^{-1}\left\langle\boldsymbol{\beta}_{m}^{u} \cdot \boldsymbol{u}_{h}, \gamma_{n}\right\rangle_{\Omega^{2}} \hat{q}_{n}, \quad \forall \gamma_{i} \in Q_{h}
$$

In multiple dimensions on parallel machines it is not practical to explicitly construct a mass matrix inverse for the $U_{h}$ space. Consequently we use an explicit third order Runge-Kutta scheme in place of the centered time integration 

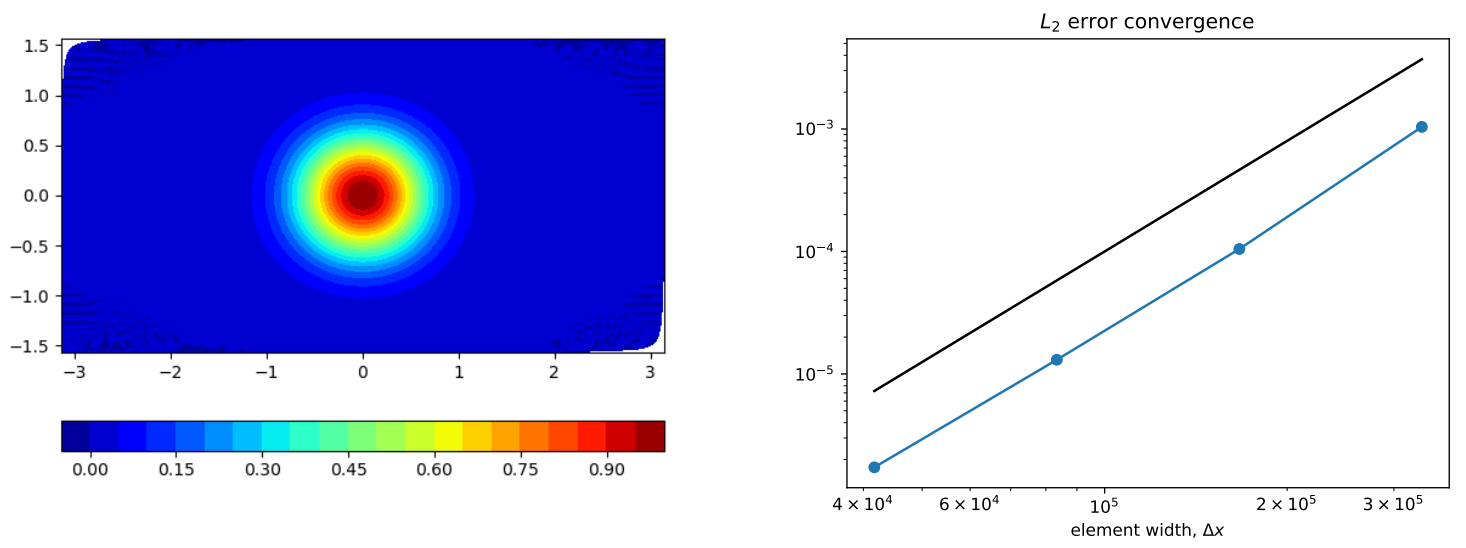

Figure 10: Solid body advection on the sphere: tracer field at final time (left) and $L_{2}$ error convergence (right). Solid line indicates a gradient of $\Delta x^{3}$.

scheme used in the previous section. This integrator takes the form [21]

$$
\begin{aligned}
q_{h}^{(1)} & =q_{h}^{n}-\Delta t y\left(\boldsymbol{u}_{h}^{n}, q_{h}^{n}\right), \\
q_{h}^{(2)} & =q_{h}^{n}-\frac{\Delta t}{4}\left(y\left(\boldsymbol{u}_{h}^{n}, q_{h}^{n}\right)+y\left(\boldsymbol{u}_{h}^{n+1}, q_{h}^{(1)}\right)\right), \\
q_{h}^{n+1} & =q_{h}^{n}-\frac{\Delta t}{6}\left(y\left(\boldsymbol{u}_{h}^{n}, q_{h}^{n}\right)+y\left(\boldsymbol{u}_{h}^{n+1}, q_{h}^{(1)}\right)+4 y\left(\boldsymbol{u}_{h}^{n+1 / 2}, q_{h}^{(2)}\right)\right),
\end{aligned}
$$

where $y\left(\boldsymbol{u}_{h}, q_{h}\right)$ represents the second term in (28).

The advection equation as described above is implemented using a cubed sphere discretisation with a physical radius of $6371220.0 \mathrm{~m}$, the details of which can be found in [12]. The first test on the sphere is one of solid body rotation, with a prescribed velocity field of $\boldsymbol{u}(\theta, \phi)=\left(20 \cos (\phi) \boldsymbol{e}_{\theta}, 0 \boldsymbol{e}_{\phi}\right)$, where $\boldsymbol{e}_{\theta}$ and $\boldsymbol{e}_{\phi}$ are the unit vectors in the zonal and meridional directions respectively, and an initial Gaussian tracer field of the form $q(t=0)=\cos (\phi) e^{-9 \theta^{2}-225 \phi^{2}}$. The test is run for a single revolution using elements of degree $p=3$ with $384 \times n_{e} / 10$ time steps, where $n_{e}$ is the number of elements in each dimension on each of the six panels of the cubed sphere, for which $n_{e}=10,20,40,80$. Figure 10 shows the final tracer distribution for $n_{e}=20$, as well as the convergence of the normalised $L^{2}$ error of the tracer field after one revolution. Using both a third order time integration scheme (29) and elements of degree $p=3$ the errors converge at third order.

The second test on the sphere is for the advection of two cosine bells within a time varying velocity field that involves both zonal transport and shear deformation, with the tracer field returning to its original position over a period of $T=12$ days. The specific details of the tracer and velocity field configurations are described in [22]. The test is run with $40 \times 40$ elements of degree $p=3$ on each face of the cubed sphere, with a time step of $\Delta t=129.6 \mathrm{~s}$.

Figures 11 and 12 show the evolution of the tracer field at days 0,6 and 12, as well as the mass conservation errors. While there is a small drift in the mass conservation errors for the one dimensional test case owing to the semi-implicit time integration scheme, as observed in Fig. 4. no drift is evident in the two dimensional advection problem, for which an explicit integration scheme (29) is used. Notably, in the absence of upwinding the tracer field becomes completely incoherent over the course of the simulation, such that the upwinded correction is essential for this test case. The solution errors do not converge at the expected rate for this test case, most likely on account of the time varying velocity field and the first order integration of the characteristics used to compute the test function nodal departure points. In order to compute these at higher order the velocity field would need to be interpolated at intermediate locations. Currently the parallel communication of data within the code is only supported along element boundaries, and not within the internal element locations needed to allow for this. 

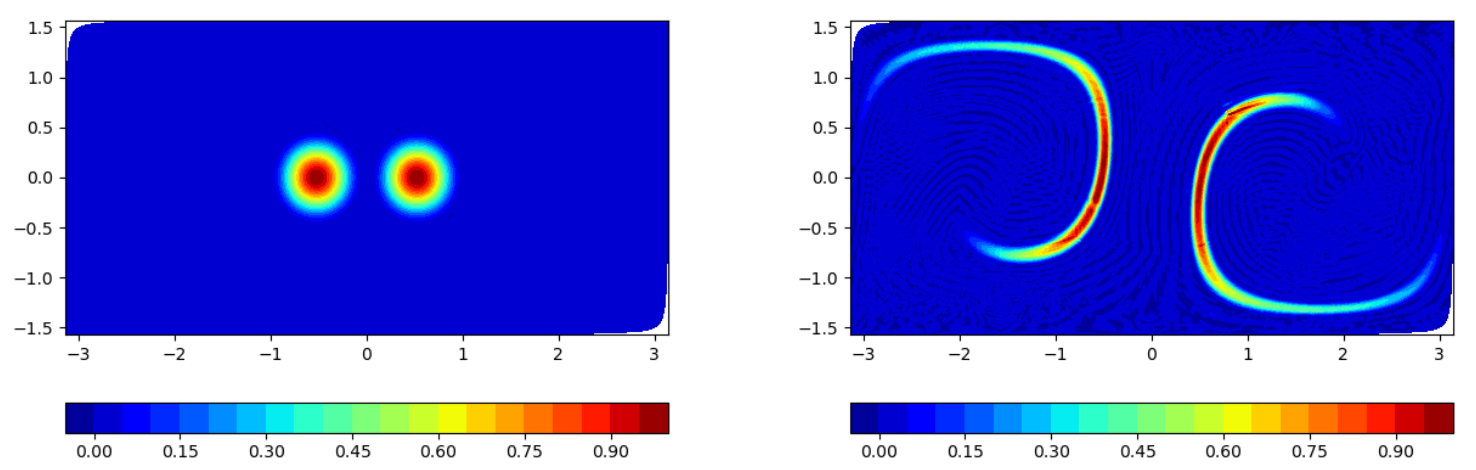

Figure 11: Deformational flow on the sphere: tracer field at days 0 (left) and 6 (right).
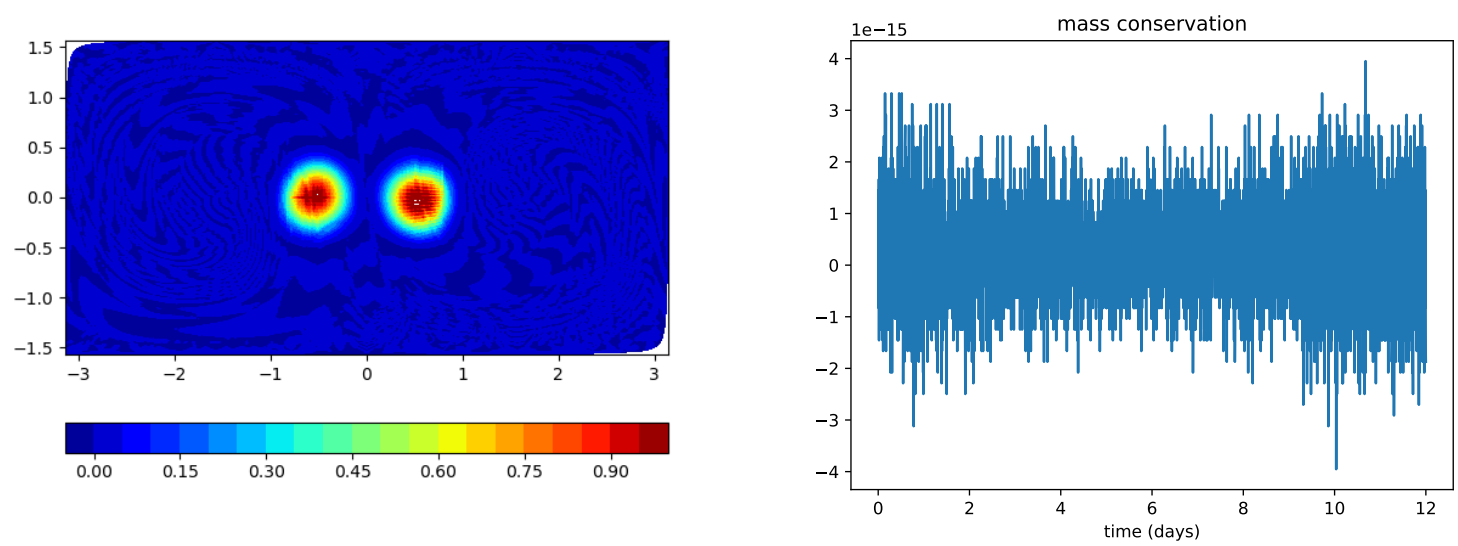

Figure 12: Deformational flow on the sphere: tracer field at day 12 (left) and mass conservation error (right). 


\section{Application to potential vorticity stabilisation in shallow water on the sphere}

In order to demonstrate the usefulness of the Petrov Galerkin upwinding described above this is applied to the diagnosis and interpolation of the potential vorticity for the rotating shallow water equations on the sphere. In additional to the discrete bases spanning the $U_{h}$ and $Q_{h}$ spaces described in the previous section (25), an additional space, $W_{h} \subset H\left(\right.$ rot, $\left.\Omega^{2}\right)$ is introduced, for which the basis functions are given as

$$
\alpha_{k}(\xi, \eta):=l_{i}(\xi) l_{j}(\eta), \quad i, j=0 \ldots p \quad k=j(p+1)+i .
$$

As well as the div compatibilty property (2), there is also a compatible mapping between the discrete spaces $W_{h}$ and $U_{h}$ via the rot operator. Together these give the sequence

$$
\mathbb{R} \longrightarrow W_{h} \stackrel{\nabla^{\perp}}{\longrightarrow} U_{h} \stackrel{\nabla \cdot}{\longrightarrow} Q_{h} \longrightarrow 0 .
$$

Since the bases for the $W_{h}$ space are $C^{0}$ continuous in both dimensions, the downwinded variant is given for $\left(\xi^{u}, \eta^{u}\right)$ as defined in (22) as

$$
\alpha_{k}^{d}(\xi, \eta):=l_{i}\left(\xi^{u}\right) l_{j}\left(\eta^{u}\right), \quad i, j=0 \ldots p \quad k=j(p+1)+i .
$$

In addition to the mixed mimetic spectral element spatial discretisation [12], the model uses a semi-implicit time integration scheme [6], that together allow for the exact balance of energy exchanges, and the exact conservation of mass, vorticity and energy in space and time. No dissipation is applied to the model, except that in the second simulation the upwinded stabilisation is applied by sampling the potential vorticity field trial functions at downstream locations in a two dimensional analogue of (21). Just as there is an adjoint relation between the discrete div and grad operators [11, a similar adjoint relationship exists between the discrete rot and curl operators [5, 14]. The downwinded potential vorticity field is therefore diagnosed in the discrete weak form analogue of $q=(\nabla \times \boldsymbol{u}+f) / h$, where $q$ is the potential vorticity, $f$ is the Coriolis term and $h$ is the fluid depth, as

$$
\left\langle\alpha_{i}, h_{h} \alpha_{j}^{d}\right\rangle_{\Omega^{2}} \hat{q}_{j}^{P G}=-\left(\mathrm{E}_{k, j}^{1,0}\right)^{\top}\left\langle\boldsymbol{\beta}_{k}, \boldsymbol{\beta}_{l}\right\rangle_{\Omega^{2}} \hat{u}_{l}+\left\langle\alpha_{i}, \alpha_{j}\right\rangle_{\Omega^{2}} \hat{f}_{j}, \quad \forall \alpha_{i} \in W_{h},
$$

where $\mathbf{E}^{1,0}$ is the incidence matrix representing the discrete strong form rot operator [12, 14]. The upwinded potential vorticity is then coupled to the shallow water system through the vector invariant form of the momentum equation $\dot{\boldsymbol{u}}=-q \times \boldsymbol{M}-\nabla \Phi$, where $\boldsymbol{M}=h \boldsymbol{u}$ is the mass flux and $\Phi=|u|^{2} / 2+g h$ is the Bernoulli function and $\dot{h}=-\nabla \cdot \boldsymbol{M},[12]$ for all $\beta_{i} \in U_{h}, \gamma_{i} \in Q_{h}$ as

$$
\left[\begin{array}{c}
\left\langle\boldsymbol{\beta}_{i}, \boldsymbol{\beta}_{j}\right\rangle_{\Omega^{2}} \hat{u}_{j}^{n+1} \\
\left\langle\gamma_{i}, \gamma_{j}\right\rangle_{\Omega^{2}} \hat{h}_{j}^{n+1}
\end{array}\right]=\left[\begin{array}{c}
\left\langle\boldsymbol{\beta}_{i}, \boldsymbol{\beta}_{j}\right\rangle_{\Omega^{2}} \hat{u}_{j}^{n} \\
\left\langle\gamma_{i}, \gamma_{j}\right\rangle_{\Omega^{2}} \hat{h}_{j}^{n}
\end{array}\right]-\Delta t\left[\begin{array}{cc}
\left\langle\boldsymbol{\beta}_{i}, \bar{q}_{h}^{d} \times \boldsymbol{\beta}_{k}\right\rangle_{\Omega^{2}} & -\left(\mathrm{E}_{l, i}^{2,1}\right)^{\top}\left\langle\gamma_{l}, \gamma_{m}\right\rangle_{\Omega^{2}} \\
\left\langle\gamma_{i}, \gamma_{j}\right\rangle_{\Omega^{2}} \mathrm{E}_{j, k}^{2,1} & 0
\end{array}\right]\left[\begin{array}{l}
\hat{\bar{M}}_{k} \\
\hat{\Phi}_{m}
\end{array}\right]
$$

where

$$
\bar{q}_{h}^{d}=\frac{1}{2} \sum_{i}\left(\alpha_{i}^{d ; n} \hat{q}_{i}^{P G ; n}+\alpha_{i}^{d ; n+1} \hat{q}_{i}^{P G ; n+1}\right)
$$

is a time centered approximation to the upwinded potential vorticity, $q_{h}^{d}$, and $\overline{\hat{M}}_{h}, \overline{\hat{\Phi}}_{h}$ are the degrees of freedom of the discrete mass flux and Bernoulli function exactly integrated between time levels $n$ and $n+1$ [6, 23]. Note that the block matrix in (34) is skew-symmetric, and so energy conservation is satisfied by the pre-multiplication of both sides by $\left[\overline{\hat{M}}_{h}^{\top} \overline{\hat{\Phi}}_{h}^{\top}\right.$ ], in analogy to the conservation of energy for the skew-symmetric advection equation $(16)$.

Since the potential vorticity, $q_{h} \in W_{h}$ is included within the skew-symmetric block matrix, the upwinding modification of the potential vorticity does not affect the conservation properties of the model. While the upwinding of the potential vorticity does dissipate potential enstrophy, this is not conserved in the original formulation due to the use of inexact quadrature [14]. This upwinded potential vorticity formulation is conceptually similar to the anticipated potential vorticity method [24], which similarly stabilises the potential vorticity by dissipating enstrophy. Note that this vorticity stabilisation does not address nonlinearities associated with the oscillation of gravity waves which may still yield aliasing errors, and also that the upwinding scheme presented here is not strictly monotone, so some of these 

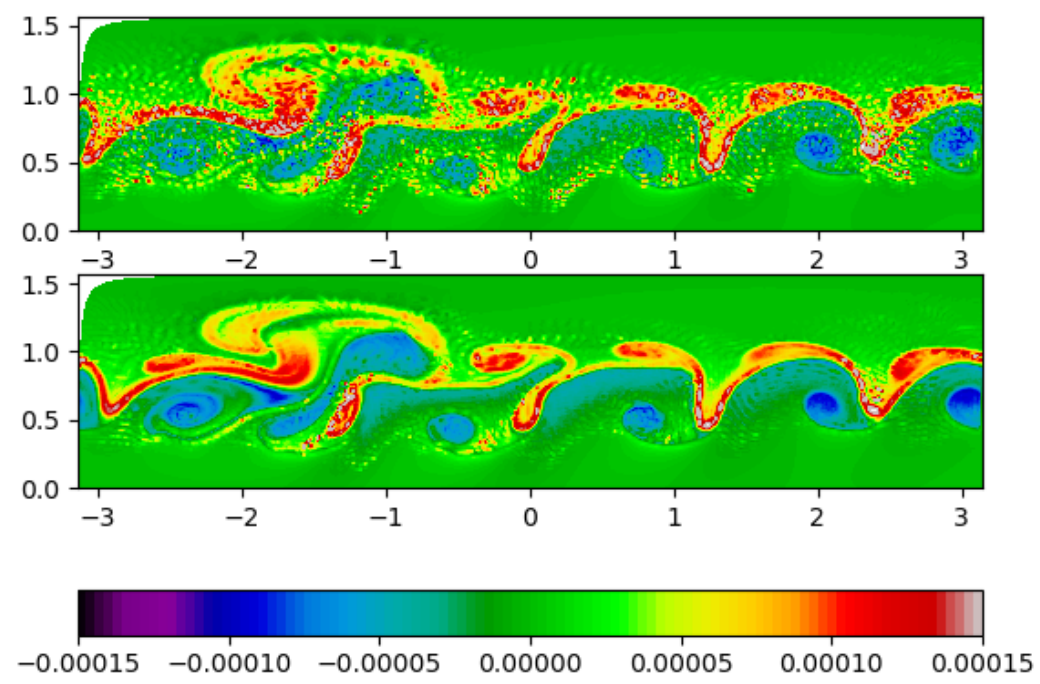

Figure 13: Vorticity field for the Galewsky test case (day 7); top: original formulation, bottom: Petrov-Galerkin upwinded formulation. Only the northern hemisphere is shown.

oscillations may still derive from the rotational term in [34). In recent work [6] a method for determining a secondary velocity reconstruction within the discrete form of the skew-symmetric operator has been presented, so as to upwind the potential gradients and mass fluxes associated with the generation of gravity waves without dissipating energy.

The code was configured according to a standard test case for rotating shallow water on the sphere [25], which is initialised as a steady jet overlaid with a small perturbation of the height field. Over several days this acts to destabilise the jet into a series of barotropic eddies. In each case the model was run with 32 elements of degree $p=3$ on each face of the cubed sphere and a time step of $\Delta t=120 \mathrm{~s}$. As observed in Fig. 13 , the Petrov-Galerkin upwinding of equation (33) leads to a more coherent solution, with fewer aliasing errors.

Figure 14 shows the vorticity, $\omega$, conservation errors integrated over the sphere. These are un-normalised, since the total vorticity integrates to zero, and integrated over the sphere in physical units, such that truncation errors may be non-negligible. There is no perceivable difference in the errors between the two schemes, and these are consistent with previous results [12]. This figure also shows the kinetic energy spectra of the two schemes at day 7 . These are computed by first interpolating the kinetic energy onto a regular latitude-longitude grid, and then decomposing this solution into spherical harmonics. Both schemes exhibit an upscale cascade of $k^{-3}$, consistent with the theory of two dimensional turbulence, however the upwinded scheme shows less oscillation in this cascade, reflecting the minimisation of aliasing errors.

\section{Conclusions}

This article describes the formulation of upwinded advection operators for mixed mimetic spectral elements in both flux form and material form. These operators exhibit dissipation of high wave numbers which suppresses spurious high frequency oscillations for sharp, poorly resolved gradients. The dissipation profiles increase with polynomial degree in a way that reflects the profile of higher order viscosity terms. Moreover the upwinded operators seal up the spectral gaps observed in dispersion relations for high order finite element methods. These upwinded formulations are relatively simple to compute and cheap to assemble. 

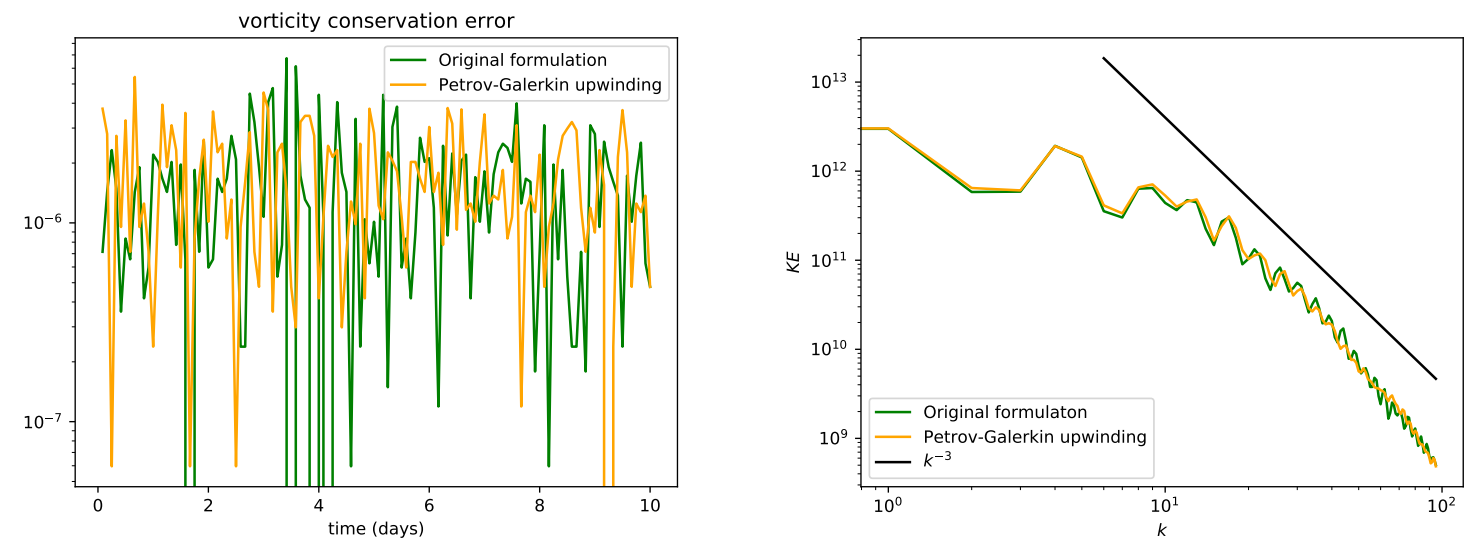

Figure 14: Vorticity conservation errors (un-normalised) for the original and upwinded formulations (left), and kinetic energy power spectra at day 7 (right).

This upwinding formulation has been coupled to an existing solver for the shallow water equations on the sphere, where it is shown to reduce aliasing errors, as well as to improve the turbulence profile. In future work the application of this scheme to the stabilisation of temperature fluxes for the compressible Euler equations will be investigated.

The scripts used to generate the one dimensional results in Section 3 can be obtained from the author's Github page at https://github.com/davelee2804/MiMSEM/tree/master/adv_eqn_1d, while those for the two dimensional test cases are found at https://github.com/davelee2804/MiMSEM/tree/hevi/sandbox/src.

\section{Appendix: computation of eigenvalues}

Assuming exact time integration, the semi-discrete advection equation is expressed as

$$
\mathbf{M}^{-1} \mathbf{A} q_{h}=\omega q_{h}
$$

for $q_{h}(x, t)=q_{h}(x) e^{-\omega t}$. The eigenvalues, $\omega_{h}$ and eigenvectors, $v_{h}$ of this square operator may be computed using any standard eigenvalue solver as $\omega_{h}, v_{h}=\operatorname{eig}\left(\mathbf{M}^{-1} \mathbf{A}\right)$ (and similarly for $\mathbf{A}_{P G ; \Delta t}$ and $-\mathbf{A}_{P G ;-\Delta t}^{\top}$ ). We then define two additional operators, an interpolation operator between degrees of freedom in $Q_{h}$ and physical coordinates, $\mathbf{Q}$, for which

$$
\mathrm{Q}_{j k}:=e_{k}\left(\xi_{j}\right)
$$

(for which the Jacobian terms cancel), and a Fourier interpolation operator, $\mathbf{F}$, for which

$$
\mathrm{F}_{j k}:=\cos \left(2 \pi k x_{j} / L\right)+i \sin \left(2 \pi k x_{j} / L\right)
$$

where $L$ is the domain length, $i$ denotes the imaginary number, and $k$ a given Fourier mode. Each eigenvector, $v_{h}$ is then projected onto a vector $v_{h}^{f}$ representing an expansion over Fourier modes as

$$
v_{h}^{f}=\mathbf{F}^{-1} \mathbf{Q} v_{h} .
$$

We then sort $v_{h}^{f}$ in order to determine the Fourier mode with the largest amplitude, $k_{h}$. This mode is then paired with the original eigenvalue, $\omega_{h}$ in order to construct the dispersion relation.

\section{Acknowledgments}

David Lee would like to thank Dr. Darren Engwirda for the idea of applying the Petrov-Galerkin scheme to the problem of potential vorticity advection in geophysical flows. This project was supported by resources and expertise provided by CSIRO IMT Scientific Computing. 


\section{References}

[1] A. N. Brooks, T. J. R. Hughes, Streamline upwind/Petrov-Galerkin formulations for convection dominated flows with particular emphasis on the incompressible Navier-Stokes equations, Comput. Meth. Appl. Mech. Engrg. 32 (1982) 199-259

[2] T. J. R. Hughes, G. R. Feijoo, L. Mazzei, J.-B. Quincy, The variational multiscale method-a paradigm for computational mechanics, Comput. Methods Appl. Mech. Engrg. 166 (1998) 3-24

[3] S. Marras, J. F. Kelly, F. X. Giraldo, M. Vázquez, Variational multiscale stabilization of high-order spectral elements for the advectiondiffusion equation, J. Comput. Phys. 231 (2012) 7187-7213

[4] M. Gerritsma, Edge functions for spectral element methods, in: Spectral and high order methods for partial differential equations, Lecture Notes in Computational Science and Engineering, Springer 76 (2011) 199-207

[5] J. Kreeft, M. Gerritsma, Mixed mimetic spectral element method for Stokes flow: A pointwise divergence-free solution, J. Comp. Phys. 240 (2013) 284-309.

[6] G. A. Wimmer, C. J. Cotter, W. Bauer, Energy conserving upwinded compatible finite element schemes for the rotating shallow water equations, J. Comput. Phys. 401 (2020) 109016

[7] T. F. Russell, M. A. Celia, An overview of research on Eulerian-Lagrangian localised adjoint methods (ELLAM), Adv. Water Resour. 25 (2002) 1215-1231

[8] W. Guo, R. D. Nair, J-M. Qiu, A conservative semi-Lagrangian discontinuous Galerkin scheme on the cubed sphere, Mon. Wea. Rev. 142 (2014) 457-475

[9] D. Lee, R. Lowrie, M. Petersen, T. Ringler, M. Hecht, A high order characteristic discontinuous Galerkin scheme for advection on unstructured meshes, J. Comput. Phys. 324 (2016) 289-302

[10] P. A. Bosler, A. M. Bradley, M. A. Taylor, Conservative Multimoment Transport along Characteristics for Discontinuous Galerkin Methods, SIAM J. Sci. Comput. 41(4) (2019) B870-B902

[11] M. E. Rognes, R. C. Kirby, A. Logg, Efficient Assembly of $H$ (div) and $H$ (curl) Conforming Finite Elements, SIAM J. Sci. Comput. 31 (2010) 4130-4151

[12] D. Lee, A. Palha, A Mixed mimetic spectral element model of the rotating shallow water equations on the cubed sphere, J. Comput. Phys. 375 (2018) 240-262

[13] A. Palha, P.P. Rebelo, M. Gerritsma, Mimetic spectral element advection, Spectral and High Order Methods for Partial Differential Equations ICOSAHOM 2012, Lecture Notes in Computational Science and Engineering, 95, Springer International Publishing (2014) 325-335

[14] D. Lee, A. Palha, M. Gerritsma, Discrete conservation properties for shallow water flows using mixed mimetic spectral elements, J. Comput. Phys. 357 (2018) 282-304

[15] M. Gerritsma, P. Bochev, A spectral mimetic least-squares method for the Stokes equations with no-slip boundary conditions, Comput. Math. Appl. 71 (2016) 2285-2300

[16] Y. Morinishi, Skew-symmetric form of convective terms and fully conservative finite difference schemes for variable density low-Mach number flows, J. Comput. Phys. 229 (2010) 276-300

[17] A. Palha, M. Gerritsma, A mass, energy, enstrophy and vorticity conserving (MEEVC) mimetic spectral element discretization for the 2D incompressible NavierStokes equations, J. Comput. Phys. 328 (2017) 200-220

[18] T. Melvin, A. Staniforth, J. Thuburn, Dispersion analysis of the spectral element method, Q. J. R. Meteorol. Soc. 138 (2012) 1934-1947

[19] C. Eldred, D. Y. Le Roux, Dispersion analysis of compatible Galerkin schemes for the 1D shallow water model, J. Comput. Phys. 371 (2018) 779-800

[20] P. A. Ullrich, D. R. Reynolds, J. E. Guerra, M. A. Taylor, Impact and importance of hyperdiffusion on the spectral element method: A linear dispersion analysis, J. Comput. Phys. 375 (2018) 427-446

[21] D. R. Durran, Numerical Methods for Fluid Dynamics: With Applications in Geophysics, Springer (2010)

[22] P. H. Lauritzen, W. C. Skamarock, M. J. Prather, M. A. Taylor, A standard test case suite for two-dimensional linear transport on the sphere, Geosci. Model. Dev. 5 (2012) 887-901

[23] D. Lee, An energetically balanced, quasi-Newton integrator for non-hydrostatic vertical atmospheric dynamics, arXiv:2004.14090 (2020)

[24] R. Sadourny, C. Basdevant, Parameterization of Subgrid Scale Barotropic and Baroclinic Eddies in Quasi-geostrophic Models: Anticipated Potential Vorticity Method, J. Atmos. Sci. 42 (1985) 1353-1363

[25] J. Galewsky, R. K. Scott, L. M. Polvani, An initial-value problem for testing numerical models of the global shallow water equations, Tellus 56A (2004) 429-440 